\title{
Molecular characterization of two recombinant isolates of telosma mosaic virus infecting Passiflora edulis from Fujian Province in China
}

\author{
Lixue Xie ${ }^{1}$, Fangluan Gao ${ }^{\text {Corresp., }}{ }^{2}$, Jianguo Shen ${ }^{3}$, Xiaoyan Zhang ${ }^{1}$, Shan Zheng ${ }^{1}$, Lijie Zhang ${ }^{1}$, Tao Li ${ }^{\text {Corresp. } 1}$ \\ ${ }^{1}$ Fruit Research Institute, Fujian Academy of Agricultural Sciences, Fuzhou, China \\ 2 Institute of Plant Virology, Fujian Agriculture and Forestry University, Fuzhou, China \\ 3 Fujian key laboratory for technology research of inspection and quarantine, Technology Center of Fuzhou Customs District, Fuzhou, China \\ Corresponding Authors: Fangluan Gao, Tao Li \\ Email address: Raindy@fafu.edu.cn, leetao06@163.com
}

Telosma mosaic virus (TeMV) is an important plant virus causing considerable economic losses to passion fruit (Passiflora edulis) production worldwide, including China. In this study, the complete genome sequence (excluding the poly(A) tail) of two TeMV isolates, Fuzhou and Wuyishan, were determined to be 10,050 and 10,057 nucleotides, respectively. Sequence analysis indicated that Fuzhou and Wuyishan isolates share 78\%-98\% nucleotide and 83\%-99\% amino acid sequence identities with two TeMV isolates of Hanoi and GX, and a proposed new potyvirus, tentatively named PasFru. Phylogenetic analysis indicated that these TeMV isolates and PasFru were clustered into a monophyletic clade with high confidences. This indicated that PasFru and the four TeMV isolates should be considered as one potyvirus species. Two recombination breakpoints were identified within the $\mathrm{Cl}$ and $\mathrm{Nlb}$ genes of the Fuzhou isolate, and also within the P1 gene of the Wuyishan isolate. To the best of our knowledge, this is the first report of TeMV recombinants worldwide. 
1 Molecular characterization of two recombinant isolates of telosma mosaic

2 virus infecting Passiflora edulis from Fujian Province in China

3

4 Lixue Xie ${ }^{1}$, Fangluan $\mathbf{G a o}^{2}$, Jianguo Shen ${ }^{3}$, Xiaoyan Zhang ${ }^{1}$, Shan Zheng ${ }^{1}$, Lijie Zhang ${ }^{1}$, 5 Tao $\mathbf{L i}^{1}$

${ }^{1}$ Fruit Research Institute, Fujian Academy of Agricultural Sciences, Fuzhou 350013, P.R. China

${ }^{2}$ Institute of Plant Virology, Fujian Agriculture and Forestry University, Fuzhou, 350002, P.R. China

${ }^{3}$ Fujian key laboratory for technology research of inspection and quarantine, Technology Center of Fuzhou Customs District, Fuzhou 350001, P.R. China

\section{Corresponding authors:}

Fangluan Gao, raindy@fafu.edu.cn

Tao Li, 1eetao06@163.com 
19 Abstract

20 Telosma mosaic virus (TeMV) is an important plant virus causing considerable economic losses

21 to passion fruit (Passiflora edulis) production worldwide, including China. In this study, the

22 complete genome sequence (excluding the poly(A) tail) of two TeMV isolates, Fuzhou and

23 Wuyishan, were determined to be 10,050 and 10, 057 nucleotides, respectively. Sequence

24 analysis indicated that Fuzhou and Wuyishan isolates share 78\%-98\% nucleotide and 83\%-99\%

25 amino acid sequence identities with two TeMV isolates of Hanoi and GX, and a proposed new

26 potyvirus, tentatively named PasFru. Phylogenetic analysis indicated that these TeMV isolates

27 and PasFru were clustered into a monophyletic clade with high confidences. This indicated that

28 PasFru and the four TeMV isolates should be considered as one potyvirus species. Two

29 recombination breakpoints were identified within the CI and NIb genes of the Fuzhou isolate,

30 and also within the P1 gene of the Wuyishan isolate. To the best of our knowledge, this is the

31 first report of TeMV recombinants worldwide. 
33

34

35

36

37

38

39

40

41

42

43

44

45

46

47

48

49

50

51

52

53

54

55

56

57

58

59

60

61

62

63

64

65

66

67

68

69

70

71

\section{Introduction}

Passion fruit (Passiflora edulis), originating in South America, is an important fruit crop that comprises a variety of cultivars and it is consumed globally (Ulmer \& Mac Dougal 2004). In China, passion fruit orchards are mainly located in the southern part of China such as Guangxi and Fujian provinces. However, the production of passion fruit is negatively affected by various plant diseases and insect pests, especially viruses (Amata 2011; Singh 2004). It is documented that passion fruits are susceptible to infection of more than 25 different viruses (Baker et al. 2011). Telosma mosaic virus (TeMV) is one of the dominant types of plant pathogens constraining sustainable development of passion fruit production.

TeMV is a member of the genus Potyvirus (Lefkowitz et al. 2018) and has a + ssRNA genome of about $9.7 \mathrm{~kb}$ (flanked by UTR at 5' and 3'ends) that encodes a polyprotein of $350 \mathrm{kDa}$, which is cleaved into 10 functional proteins by virus-encoded proteinases (Lefkowitz et al. 2018). In addition, a short ORF (PIPO) is translated by +2 nucleotide frame shifting within the P3 cistron and expressed as a P3-PIPO fusion product (Chung et al. 2008). TeMV was firstly reported to infect Chinese violet (Telosma cordata) in Vietnam (Ha et al. 2008), patchouli (Pogostemon cablin) in Indonesia (Noveriza et al. 2012), then passion fruit in Thailand (Chiemsombat et al. 2014). Recently this virus has also been reported to be present in China (Chen et al. 2018; Xie et al. 2017; Yang et al. 2018).

During a survey of passion orchards in 2017 in Fujian Province, China, plants exhibiting virus-like mosaic and crinkle symptoms were prevalent. The disease causes a serious reduction in production and decreases the quality of passion fruit (Xie et al. 2017). In 2018, a TeMV isolate named PasFru (accession number MG944249), collected from Haikou Province, was identified and proposed to be a new member of the Potyvirus genus based on analyses of the complete genome sequence (Yang et al. 2018). In addition to PasFru, only one complete genome of TeMV isolate (named Hanoi) from Vietnam has been deposited in GenBank (accession number of NC_009742), although TeMV has been identified in many countries. One nearly complete genome of TeMV isolate from Guangxi Province in China (named GX, accession number KJ789129) is also available in GenBank. To date, no recombinant TeMV isolate has been reported worldwide.

The objectives of this study were (i) to identify two new TeMV isolates from passion fruit in China using transmission electron microscopy, indirect ELISA and RT-PCR; (ii) obtain their complete genome sequences and characterize their genomic structure; (iii) and clarify the current confusion surrounding the taxonomic status of some of these TeMV isolates, particularly the proposed new potyvirus PasFru.

\section{Materials \& Methods}

\section{Sample collection, electron microscopy and serological detection}

Two passion fruit samples showing mosaic and crinkle symptoms on the leaves (Fig. 1A) were collected in 2017 from a commercial orchard in Fujian Province, China (Xie et al. 2017). After 
negatively staining with $2 \%$ phosphotungstic acid $(\mathrm{pH} 6.7)$, crude sap from the passion fruit sample was placed onto formvar-coated copper grids, and then examined using an H-7650 transmission electron microscope (Hitachi, Japan) operating at $80 \mathrm{kV}$. Fresh leaf samples of passion fruit were manually homogenized in pestles for homogenization with $0.05 \mathrm{M}$ sodium carbonate buffer, $\mathrm{pH}$ 9.6. The antigen-coated indirect ELISA protocol was performed by using universal potyvirus antiserum (Agdia, Elkhart, IN, USA) according to the manufacturer's instructions. All samples were tested in duplicate wells in microtiter plates. Absorbance values at $405 \mathrm{~nm}$ were measured with an automatic ELISA reader (Infinite M200, Tecan, Switzerland). Sample with absorbance value at least twice that of healthy control was considered positive.

\section{RNA extraction and cDNA synthesis}

Total RNA was extracted from the leaf tissue which was positive with virus infection by ELISA using an RNA extraction kit (Qiagen, Hilden, Germany). The quantity and quality of the extracted RNA were determined by measuring absorptions at $260 \mathrm{~nm}$ and $280 \mathrm{~nm}$ with a NanoDrop 2000c (Thermo Scientific, Massachusetts, USA). The first-strand cDNA was synthesized using Moloney murine leukemia virus (M-MLV) reverse transcriptase (Promega, Madison, Wisconsin, USA) following the manufacturer's protocol. For the reaction, in total of $11 \mu \mathrm{l}$ mixture containing $3 \mu \mathrm{l}$ RNA $(\sim 1 \mu \mathrm{g}), 1 \mu \mathrm{l}$ random primer $(100 \mu \mathrm{m})$ and $7 \mu \mathrm{l}$ DEPC-treated water were incubated at $70^{\circ} \mathrm{C}$ for $10 \mathrm{~min}$. Then, the mixture was transferred immediately to an ice bath for $5 \mathrm{~min}$. Finally, $5 \mu 15 \times$ buffer (Promega, Madison, Wisconsin, USA), $2 \mu 1 \mathrm{dNTP}$ mix (Promega, Wisconsin, USA), $1 \mu$ RNAsin Plus RNase inhibitor (Promega, Madison, Wisconsin, USA) and $1 \mu \mathrm{l}$ M-MLV reverse transcriptase (Promega, Madison, Wisconsin, USA) were added to the mixture of primer and RNA. The RT reaction was carried out at $42^{\circ} \mathrm{C}$ for $60 \mathrm{~min}$ followed by at $70^{\circ} \mathrm{C}$ for $10 \mathrm{~min}$. The cDNA was chilled on ice and stored at $-70^{\circ} \mathrm{C}$.

\section{Molecular detection of virus using polymerase chain reaction (PCR)}

For the detection of the genus Potyvirus, a set of universal potyvirus primer LegPotyF $5^{\prime}$ GCWKCHATGATYGARGCHTGGG-3' and LegPotyR 5' -AYYTGYTYMTCHCCATCCATC$3^{\prime}$ (Wylie et al., 2010) was used to amplify a fragment of approximately $680 \mathrm{bp}$. PCR reactions were performed in a $25 \mu 1$ volume containing $12.5 \mu \mathrm{L}$ of $2 \times$ Taq PCR mix (Promega, Wisconsin, USA), $1 \mu 1$ of each primer $(10 \mu \mathrm{M}), 2 \mu 1$ of cDNA, and $8.5 \mu 1$ DEPC-treated water. PCR conditions were as follows: initial denaturation at $94^{\circ} \mathrm{C}$ for $3 \mathrm{~min}$, followed by 35 cycles of denaturation at $94^{\circ} \mathrm{C}$ for $30 \mathrm{~s}$, annealing at $45^{\circ} \mathrm{C}$ for $45 \mathrm{~s}$ and an extension step at $72^{\circ} \mathrm{C}$ for $1 \mathrm{~min}$. The amplification program was followed by a final extension step at $72^{\circ} \mathrm{C}$ for $10 \mathrm{~min}$.

TeMV was detected by using specific primers TeMV-CPf 5'TCAAGTAAGGTGGATGATGTT-3' and TeMV-CPr 5'-CTGCACAGAGCCAACCCCAA-3' as previously described (Xie et al., 2017). The primer pair TeMV-CPf/ TeMV-CPr was designed to amplify the full length of the coat protein $(\mathrm{CP})$ gene $(\sim 816 \mathrm{bp})$. PCR was performed in a final volume of $25 \mu \mathrm{l}$ in a reaction mixture consisting of $2.5 \mu \mathrm{L}$ of $10 \times$ PCR Buffer (TaKaRa, Dalian, 
111 China), $2.5 \mu \mathrm{l}$ of $\mathrm{MgCl}_{2}(25 \mathrm{mM}), 1 \mu \mathrm{l}$ of dNTPs $(2.5 \mathrm{mM}), 1 \mu \mathrm{l}$ of each primer $(10 \mu \mathrm{M}), 0.2 \mu 1$ of

112 Taq DNA polymerase $(5 \mathrm{U} / \mu \mathrm{L}), 3 \mu \mathrm{l}$ of cDNA, and $13.8 \mu 1$ DEPC-treated water. PCR conditions 113 were as follows: initial denaturation at $94^{\circ} \mathrm{C}$ for $3 \mathrm{~min}$, followed by 35 cycles of denaturation at $11494^{\circ} \mathrm{C}$ for $30 \mathrm{~s}$, annealing at $55^{\circ} \mathrm{C}$ for $1 \mathrm{~min}$ and an extension step at $72^{\circ} \mathrm{C}$ for $1 \mathrm{~min}$. A final 10 115 min elongation step at $72^{\circ} \mathrm{C}$ was performed at the end of the 35 cycles.

\section{Cloning and sequencing of TeMV complete genome} photographed under UV-light. The target fragments of PCR products were purified by using Agarose Gel DNA Purification Kit (TaKaRa, Dalian, China). The purified products were ligated to pMD18-T vector (TaKaRa, Dalian, China) and then transformed into the E. coli DH5 $\alpha$ competent cell. The positive clones containing the insert fragment were identified by PCR. Three of the positive clones were sequenced by Shanghai Sangon Biological Engineering Technology and Service Co., Ltd.

To amplify and clone the full-length genome sequences, five overlapping fragments covering the coding regions of the TeMV genome were amplified using RT-PCR with 5 pairs of specific primers designed from the highly conservative region of the TeMV genome (Table S1). RACE PCRs for the 5' and 3' -ends of the virus genome were conducted using the SMARTer RACE 5'/3' Kit (Clontech, Dalian, China) and 3'-Full RACE Core Set with PrimeScript ${ }^{\mathrm{TM}}$ RTase (TaKaRa, Dalian, China). Long PCR fragment was determined by using primer-walking method (Sangon Biotech, Shanghai, China). At least three colonies derived from each transformant were sequenced and the consensus sequences were used for genome assembling using DNAMAN version 9.0 program (Lynnon, Quebec, Canada). Complete genome sequences of the Fuzhou and isolate (accession number MK340754) and Wuyishan isolate (accession number MK340755) were deposited in GenBank.

\section{Phylogenetic and recombination analyses}

The complete genome was assembled from overlapping RT-PCR clones after removal of the vector and primer sequence. To identify the closest relatives of the Fuzhou and Wuyishan isolates, we performed a BLASTn search against the $\mathrm{nt} / \mathrm{nr}$ databases and a sequence identity matrix using BioEdit. The putative cleavage site patterns in the polyprotein were identified using online website (http://www.dpvweb.net/potycleavage/index.html).

To reveal the evolutionary relationship of TeMV, the reference sequences of other potyviruses were retrieved from the NCBI GenBank database. We aligned sequences (excluding the UTRs) by codon and removed poorly aligned regions using TranslatorX (Abascal et al. 2010). Maximum-likelihood-based phylogenetic analysis was performed using IQ-TREE 1.6.6 (Nguyen et al. 2014) under the GTR+F+R5 nucleotide substitution model, which was selected by ModelFinder (Kalyaanamoorthy et al. 2017). Topological support was estimated by 5000 Ultrafast bootstrap replicates as well as the SH-aLRT test with 1000 replicates (Guindon et al. 
150

151

152

153

154

155

156

157

158

159

160

161

162

163

164

165

166

167

168

169

170

171

172

173

174

175

176

177

178

179

180

181

182

183

184

185

186

187

188

2010).

Recombination analysis were conducted using seven different methods (RDP, GENECONV, BOOTSCAN, MAXCHI, CHIMAERA, SISCAN, and 3SEQ) implemented in the RDP4 package (Martin et al. 2015). For each putative recombinant breakpoint, a Bonferroni-corrected $p$-value cut-off of 0.01 was calculated. To reduce the presence of false positives, recombination events supported by at least four methods with an associated $p$-value of $<10^{-6}$ were considered to be significant.

\section{Results}

\section{Detection of TeMV}

The results of electron microscopy showed the presence of potyvirus-like flexuous rod particles of about 750-770 nm in length (Fig.1B). Presence of potyvirus infection was confirmed by using Indirect-ELISA. Parts of the nuclear inclusion protein b (NIb), and CP gene of TeMV (680 bp) and the entire CP gene of TeMV (816 bp) were obtained by RT-PCR (Fig. 1C).

A BLASTn search against GenBank indicated that the RT-PCR sequences obtained here share more than 98\% nucleotide identities with TeMV (accession number KJ789129). These results suggested that TeMV was present in passion fruit plants showing mosaic and crinkle leaves from Fuzhou and Wuyishan .

\section{Molecular genomic characterization of TeMV isolate}

The complete genome sequence (excluding the $3^{\prime}$ poly(A) tail) of Fuzhou is 10,050 nucleotides (nts) in length and of Wuyishan is 10,057 nts in length. Their 5' -untranslated regions (5'-UTRs) are 169 and 177 nts, respectively, while the $3^{\prime}$-UTRs were both 251 nts (Table 1). Both contained an open reading frame of $9630 \mathrm{nts}$, encoding a polyprotein of 3209 amino acids. An additional short open reading frame translated by ribosomal frameshift, called PIPO, was also identified within the P3 cistron (Chung et al. 2008); this includes the highly conserved $\mathrm{G}_{2} \mathrm{~A}_{6}$ motif at nucleotide positions 2873-3913 in the genome of the Fuzhou isolate, and at nucleotide positions 2880-3920 in the genome of the Wuyishan isolate.

The polyproteins of Fuzhou and Wuyishan were predicted to be proteolytically processed into ten mature peptides. Their cleavage sites are in consensus to those of other TeMV isolates, whose dipeptides are Y/S, G/G, Q/G, Q/S, Q/S, Q/G, E/S, Q/S and Q/S (Fig. 2). Details regarding genome organization, and protein sizes are presented in Table 1.

Pairwise comparisons showed that Fuzhou shares 78\%, 98\% and 96\% nucleotide sequences and $84 \%, 99 \%$ and $98 \%$ amino acid sequence identities with Hanoi, GX and PasFru, respectively at the complete genome level (Table 1A). Wuyishan shares $78 \%, 99 \%$ and $88 \%$ nucleotide sequences and $83 \%, 99 \%$ and $92 \%$ amino acid sequence identities with Hanoi, GX, and PasFru, respectively at the complete genome level (Table 1A). At the individual cistron level, Fuzhou and Wuyishan share 43\%-100 \% nucleotide sequence identity and $40 \%-100 \%$ amino acid sequence identity with Hanoi, GX and PasFru, respectively (Table 1B). However, the CP of 
189 Fuzhou and Wuyishan both share more than $85.9 \%$ nucleotide and $88.9 \%$ amino acid sequence

195

196

197

198

199

200

201

202

203

204

205

206

207

208

209

210

211

212

213

214

215

216

217

identities with Hanoi, GX and PasFru, respectively. According to the accepted species demarcation criterion for the genus Potyvirus, Fuzhou, Wuyishan, Hanoi, GX and PasFru should be considered as one species of Potyvirus. Interestingly, the N-terminal region of P1 sequences of Fuzhou and Wuyishan are divergent to both length and amino acid sequences, especially with Hanoi (Fig. 2B).

\section{Phylogenetic classification of TeMV}

Our phylogenetic analysis indicated that Wuyishan and Fuzhou were clustered into a monophyletic clade with high confidence (UFBoot/ BPs=100), together with GX, Hanoi and PasFru (Fig. 3), suggesting that these isolates share a common ancestral origin. Notably, PasFru was not placed in a new taxon, although it was proposed to be a new member of the Potyvirus species (Yang et al. 2018). Hanoi, isolated from Telosma cordata in Vietnam, was clustered in an outer branch of the other TeMV isolates collected from Passiflora edulis in China.

\section{Recombination signals in TeMV}

Recombination analyses identified a recombination region of approximately 4, 000-nucleotides in the Fuzhou genome, with two recombination breakpoints within the CI and NIb regions (Fig. 3B), and PasFru and GX isolates were identified as its parents with a high level of significance by the RDP suite (GENECONV, $\mathrm{p} \leq 7.70 \mathrm{E} \times 10^{-134}$, Bootscan, $\mathrm{p} \leq 1.34 \times 10^{-138}$; Maxchi, $\mathrm{p} \leq 2.19 \times 10^{-}$ 45; Chimaera, $\mathrm{p} \leq 5.65 \times 10^{-47}$; Siscan, $\mathrm{p} \leq 7.25 \times 10^{-54}$ and 3 Seq , $\mathrm{p} \leq 4.44 \times 10^{-15}$ ) (Fig. $3 \mathrm{C}$ ). A short recombination region within the $\mathrm{P} 1$ region was identified in the Wuyishan genome (Fig. 3B), and PasFru and Hanoi isolates were recognized as its parents with high degree of support by RDP $\left(\mathrm{p} \leq 5.53 \times 10^{-6}\right)$, Bootscan $\left(\mathrm{p} \leq 5.21 \times 10^{-23}\right)$, Maxchi $\left(\mathrm{p} \leq 4.12 \times 10^{-18}\right)$, Chimaera $\left(\mathrm{p} \leq 2.13 \times 10^{-10}\right)$, Siscan $\left(\mathrm{p} \leq 5.92 \times 10^{-9}\right)$ and 3 Seq $\left(\mathrm{p} \leq 1.11 \times 10^{-14}\right)$ (Fig. 3C).

\section{Discussions}

Recombination is an important evolutionary factor that generated genetic variation in viral populations. The phenomenon is prevalent in potyviruses, e.g., in turnip mosaic virus (Yasaka et al. 2015), potato virus Y (Hu et al. 2009), and Ornithogalum mosaic virus (Gao et al. 2018). Among the potyviruses infecting passion fruit, TeMV has been a prevailing virus throughout southern China in recent years (Chen et al. 2018; Xie et al. 2017; Yang et al. 2018). However, the recombinant TeMV isolate has not yet been investigated up to date. In the current study, the complete genomes of two isolates of TeMV were fully sequenced from passion fruit plants in Fujian, China. We provided for the first-time evidence that intra-species recombination has occurred in the genomes of Fuzhou and Wuyishan isolates. One possible explanation is that the recombinant isolates generally possess a fitness advantage over nonrecombinants (Quenouille et al. 2013). Vegetatively propagated crops are particularly susceptible to viral infection (Kraus et al., 2008). As passion fruit cultivation relies heavily on vegetative propagation, the imported 
228

229

230

231

232

233

234

235

236

237

238

239

240

241

242

243

244

245

246

247

248

249

250

251

252

253

254

255

256

257

258

259

260

261

262

263

264

265

passion fruit may certainly carry a risk of introduction of passion fruit pathogens, including the new recombinant TeMV isolates, prompting us to pay more attention to their transmission in the production of passion fruit.

In the family Potyviridae, the species demarcation criteria for the complete ORF are less than $76 \%$ nucleotide and $82 \%$ amino acid identity. The thresholds for species demarcation are $<$ $58 \%$ nucleotide identity for the $\mathrm{P} 1$ coding region, and $<74 \%-78 \%$ nucleotide identity for other coding regions. However, for the coat protein, the optimal species demarcation criteria are $<76 \%$ $77 \%$ nucleotide and $<80 \%$ amino acid identity, respectively. According to this criterion, Fuzhou, Wuyishan, GX, PasFru and Hanoi should be considered as isolates of TeMV. In Potyvirus, P1 is the most divergent protein varying in length and its amino acid sequences (Rohožková \& Navrátil 2011), which is considered to be the determinant of potyviruses adapting to a wide range of host species (Valli et al. 2007). Yang et al (2018) found molecular differences between TeMV isolate PasFru and Hanoi, particularly in the P1 coding region. A similar observation was also made for two TeMV isolates sequenced in this study - the N-terminal region of P1 sequences of Fuzhou and Wuyishan are divergent to Hanoi both in length and amino acid sequences.

The results of this study identified PasFru as the common progenitor of Fuzhou and Wuyishan. This suggested that PasFru possibly emerged more ancient than other TeMV isolates in China. However, the temporal dynamics of TeMV could not be estimated since the TeMV isolates in our study are relatively limited, particularly the earlier isolates were not sampled. In addition, TeMV tended to cluster according to their geographical or host species origin; this could be explained, in part, as geography-specific or host-driven adaptation (Xie et al. 2017). However, this possibility was not tested due to the limited number of geographic region and host species available. Further studies aiming to understand the evolutionary timescale and patterns of adaptive evolution of TeMV will be interesting based on larger data sets. These will lead to a more comprehensive view of the evolutionary history of TeMV.

\section{Conclusions}

In summary, this study represents one of serval attempts to reveal the taxonomic status of TeMV isolates. Our sequence analyses suggest that PasFru and other TeMV isolates should be considered as one potyvirus species. In addition, we found Fuzhou and Wuyishan were TeMV recombinants. To the best of our knowledge, this is the first report of TeMV recombinants worldwide.

\section{Funding}

This work was supported by the National Key R\&D Program of China (2016YFF0203200), the Agricultural Science and Technology Major Project Funds of Fujian (Grant No. 2017NZ0003-1) and the National Natural Science Foundation of China (Grant No. 31570146). 


\section{Conflict of Interest}

The authors declare that they have no conflicts of interest.

269

\section{Author Contributions}

271

- Lixue Xie performed experiments, analyzed the data, wrote the paper

- Fangluan Gao analyzed the data, wrote the paper, prepare figures and/or tables, reviewed drafts of the paper

- Jianguo Shen analyzed the data and reviewed drafts of the paper

- Xiaoyan Zhang performed experiments, prepared figures and/or tables

- Shan Zheng performed experiments and prepared figures and/or tables

- Lijie Zhang performed experiments and prepared figures and/or tables

- Tao Li wrote the paper and reviewed drafts of the paper

\section{Ethical approval}

This article does not contain any studies with human participants or animals performed by any of the authors.

\section{References}

Abascal F, Zardoya R, and Telford MJ. 2010. TranslatorX: multiple alignment of nucleotide sequences guided by amino acid translations. Nucleic Acids Res 38:W7-13. 10.1093/nar/gkq291

Amata RL. 2011. Management strategies for fungal diseases in passion fruit production systems in Kenya. Acta horticulturae:207-213.

Baker CA, Jeyaprakash A, Webster CG, and Adkins S. 2011. Viruses infecting passiflora species in Florida. European Biophysics Journal 40:175-180.

Chen S, Yu N, Yang S, Zhong B, and Lan H. 2018. Identification of telosma mosaic virus infection in Passiflora edulis and its impact on phytochemical contents. Virology Journal 15:168.

Chiemsombat P, Prammanee S, and Pipattanawong N. 2014. Occurrence of telosma mosaic virus causing passion fruit severe mosaic disease in Thailand and immunostrip test for rapid virus detection. Crop Protection 63:41-47.

Chung BY, Miller WA, Atkins JF, and Firth AE. 2008. An overlapping essential gene in the Potyviridae. Proceedings of the National Academy of Sciences of the United States of America 105:5897-5902. 10.1073/pnas.0800468105

Gao F, Du Z, Shen J, Yang H, and Liao F. 2018. Genetic diversity and molecular evolution of Ornithogalum mosaic virus based on the coat protein gene sequence. PeerJ 6:e4550. 10.7717 peerj. 4550

Gibbs A, and Ohshima K. 2010. Potyviruses and the digital revolution. Annu Rev Phytopathol 48:205-223. 10.1146/annurev-phyto-073009-114404

Guindon S, Dufayard JF, Lefort V, Anisimova M, Hordijk W, and Gascuel O. 2010. New algorithms and 
methods to estimate maximum-likelihood phylogenies: assessing the performance of PhyML 3.0. Syst Biol 59:307-321. 10.1093/sysbio/syq010

Ha C, Coombs S, Revill PA, Harding RM, Vu M, and Dale JL. 2008. Design and application of two novel degenerate primer pairs for the detection and complete genomic characterization of potyviruses. Arch Virol 153:25-36.

Hu X, Karasev AV, Brown CJ, and Lorenzen JH. 2009. Sequence characteristics of Potato virus $Y$ recombinants. Journal of General Virology 90:3033-3041.

Kraus J, Tzanetakis I E, Putnam M L, Martin R R. Complete nucleotide sequence of an isolate of coleus vein necrosis virus from verbena. Archives of Virology, 2008, 153(2):381-384.

Kalyaanamoorthy S, Minh BQ, Wong TKF, von Haeseler A, and Jermiin LS. 2017. ModelFinder: fast model selection for accurate phylogenetic estimates. Nature Methods 14:587-589. $10.1038 /$ nmeth.4285

Lefkowitz EJ, Dempsey DM, Hendrickson RC, Orton RJ, Siddell SG, and Smith DB. 2018. Virus taxonomy: the database of the International Committee on Taxonomy of Viruses (ICTV). Nucleic Acids Res 46:D708-D717. 10.1093/nar/gkx932

Martin DP, Murrell B, Golden M, Khoosal A, and Muhire B. 2015. RDP4: Detection and analysis of recombination patterns in virus genomes. Virus Evolution 1:vev003. 10.1093/ve/vev003

Nguyen L-T, Schmidt HA, von Haeseler A, and Minh BQ. 2014. IQ-TREE: A fast and effective stochastic algorithm for estimating maximum likelihood phylogenies. Mol Biol Evol 32:268-274. 10.1093/molbev/msu300

Noveriza R, Suastika G, Hidayat SH, and Kartosuwondo U. 2012. Potyvirus associated with mosaic disease on patchouli (Pogostemon cablin (Blanco) Benth.) plants in Indonesia. Journal of ISSAAS (International Society for Southeast Asian Agricultural Sciences) 18:131-146.

Quenouille, J., Vassilakos, N., Moury, B., 2013. Potato virus Y: a major crop pathogen that has provided major insights into the evolution of viral pathogenicity. Molecular plant pathology 14, 439-452.

Rohožková J, and Navrátil M. 2011. P1 peptidase - a mysterious protein of family Potyviridae. Journal of Biosciences 36:189-200. 10.1007/s12038-011-9020-6

Singh SJ. 2004. Virus and phytoplasma diseases of passion fruit. In: Naqvi SAMH (eds) Diseases of Fruits and Vegetables: Volume II Springer, Dordrecht.

Ulmer T, and Mac Dougal JM. 2004. Passiflora: Passionflowers of the world. Portland: Timber Press.

Valli A, López-Moya JJ, and García JA. 2007. Recombination and gene duplication in the evolutionary diversification of P1 proteins in the family Potyviridae. Journal of General Virology 88:10161028. 10.1099/vir.0.82402-0

Xie L, Zhang X, Shan Z, Zhang L, and Tao L. 2017. Molecular identification and specific detection of telosma mosaic virus infecting passion fruit. Scientia Agricultura Sinica 50:4725-4734.

Yang K, Yan H, Song L, Jin P, Miao W, and Cui H. 2018. Analysis of the complete genome sequence of a potyvirus from passion fruit suggests its taxonomic classification as a member of a new species. Arch Virol 13:1-4.

Yasaka R, Ohba K, Schwinghamer MW, Fletcher J, Ochoa-Corona FM, Thomas JE, Ho SY, Gibbs AJ, 
and Ohshima K. 2015. Phylodynamic evidence of the migration of turnip mosaic potyvirus from

Europe to Australia and New Zealand. Journal of General Virology 96:701-713. 
349 Table 1 (A) Percentage of nucleotide (below diagonal) and amino acids (above diagonal) sequence 350 identities of the complete genome among telosma mosaic virus (TeMV) isolates. (B) Percentage

351 nucleotide and amino acids (in parentheses) identities of the untranslated and protein-coding regions of

352 Fuzhou and Wuyishan to other TeMV genomes, for which (nearly) complete genome sequences were 353 available.

\begin{tabular}{|c|c|c|c|c|c|c|}
\hline Virus isolate & Fuzhou & \multicolumn{2}{|c|}{ Hanoi } & GX & PasFru & Wuyishan \\
\hline \multicolumn{7}{|l|}{ (A) } \\
\hline Fuzhou & - & \multicolumn{2}{|c|}{84} & 99 & 98 & 93 \\
\hline Hanoi & 78 & \multicolumn{2}{|c|}{-} & 87 & 84 & 83 \\
\hline GX & 98 & \multicolumn{2}{|c|}{79} & - & 97 & 99 \\
\hline PasFru & 96 & \multicolumn{2}{|c|}{78} & 92 & - & 92 \\
\hline Wuyishan & 92 & 78 & & 99 & 88 & - \\
\hline \multirow{2}{*}{ Segment } & \multirow{2}{*}{$\begin{array}{l}\text { Genome Position } \\
\text { (Fuzhou/Wuyishan) }\end{array}$} & \multicolumn{2}{|c|}{ Protein size } & \multicolumn{3}{|c|}{ Sequence identity } \\
\hline & & aa & $\mathrm{kDa}$ & GX & PasFru & Hanoi \\
\hline \multicolumn{7}{|l|}{ (B) } \\
\hline $5^{\prime}-\mathrm{UTR}$ & $1-169 / 1-177$ & - & - & - & $99 / 100$ & $70 / 93$ \\
\hline P1 & $170-1501 / 178-1508$ & 444 & 50 & - & $\begin{array}{l}91 / 62.2 \\
(90 / 61)\end{array}$ & $\begin{array}{l}47 / 43 \\
(45 / 40)\end{array}$ \\
\hline HC-pro & $1502-2872 / 1509-2879$ & 457 & 52 & - & $\begin{array}{l}99 / 88 \\
(99 / 94)\end{array}$ & $\begin{array}{l}74 / 75 \\
(82 / 82)\end{array}$ \\
\hline P3 & $2873-3913 / 2880-3920$ & 347 & 41 & $\begin{array}{l}58 / 63 \\
(59 / 63)\end{array}$ & $\begin{array}{l}98 / 90 \\
(99 / 93)\end{array}$ & $\begin{array}{l}70 / 70 \\
(66 / 66)\end{array}$ \\
\hline PIPO & $3325-3558 / 3332-3565$ & 76 & 8.7 & $\begin{array}{l}97 / 97 \\
(95 / 100)\end{array}$ & $\begin{array}{l}99 / 99 \\
(99 / 96)\end{array}$ & $\begin{array}{l}81 / 81 \\
(68 / 71)\end{array}$ \\
\hline $6 \mathrm{~K} 1$ & $3914-4069 / 4050-4076$ & 52 & 6 & $\begin{array}{l}96 / 100 \\
(100 / 100)\end{array}$ & $\begin{array}{l}99 / 96 \\
(100 / 100)\end{array}$ & $\begin{array}{l}79 / 79 \\
(85 / 85)\end{array}$ \\
\hline $\mathrm{CI}$ & $4070-5971 / 4077-5978$ & 634 & 71 & $\begin{array}{l}99 / 100 \\
(100 / 100)\end{array}$ & $\begin{array}{l}91 / 90 \\
(99 / 99)\end{array}$ & $\begin{array}{l}80 / 80 \\
(93 / 93)\end{array}$ \\
\hline $6 \mathrm{~K} 2$ & $6972-6130 / 5979-6137$ & 53 & 6 & $\begin{array}{l}100 / 99 \\
(100 / 100)\end{array}$ & $\begin{array}{l}84 / 84 \\
(92 / 92)\end{array}$ & $\begin{array}{l}74 / 74 \\
(85 / 85)\end{array}$ \\
\hline $\mathrm{VPg}$ & $6131-6700 / 6138-6707$ & 190 & 22 & $\begin{array}{l}100 / 100 \\
(100 / 100)\end{array}$ & $\begin{array}{l}94 / 93 \\
(92 / 92)\end{array}$ & $\begin{array}{l}75 / 75 \\
(85 / 85)\end{array}$ \\
\hline $\mathrm{NIa}$ & $6701-7429 / 6708-7436$ & 243 & 28 & $\begin{array}{l}100 / 100 \\
(100 / 100)\end{array}$ & $\begin{array}{l}93 / 93 \\
(95 / 95)\end{array}$ & $\begin{array}{l}77 / 77 \\
(84 / 84)\end{array}$ \\
\hline $\mathrm{NIb}$ & $7430-8980 / 7437-8987$ & 517 & 60 & $\begin{array}{l}98 / 100 \\
(100 / 100)\end{array}$ & $\begin{array}{l}94 / 92 \\
(96 / 96)\end{array}$ & $\begin{array}{l}81 / 81 \\
(87 / 87)\end{array}$ \\
\hline $\mathrm{CP}$ & $8981-9796 / 8988-9803$ & 272 & 31 & $\begin{array}{l}96 / 100 \\
(97 / 99)\end{array}$ & $\begin{array}{l}99 / 96 \\
(100 / 97)\end{array}$ & $\begin{array}{l}86 / 86 \\
(90 / 89)\end{array}$ \\
\hline $3^{\prime}$-UTR & $9800-10050 / 9807-10057$ & - & - & $99 / 98$ & $99 / 98$ & $91 / 90$ \\
\hline
\end{tabular}


354

355

356

357

358

359

360

361

362

363

364

365

366

367

368

369

370

371

372

373

374

375

376

377

378

379

380

381

382

383

384

\section{Figure Legends}

Fig. 1 Identification of $P$. edulis leaves infected with telosma mosaic virus (TeMV). (A). Associated disease symptoms on passion fruit infected with TeMV isolate of Fuzhou. (B). Transmission electron micrographs of virions from crude extracts of $P$. edulis infected with TeMV. (C). RT-PCR amplification of partial TeMV NIb (left) and CP (right) genes. The fragments are separated in agarose gel electrophoresis. 100bp DNA ladder (lane M). TeMV isolates of Fuzhou and Wuyishan (lanes 1-2), and negative control (lanes 3-4).

Fig. 2 (A) Comparisons between the predicted protease cleavage sites of telosma mosaic virus (TeMV) isolates. Letters in bold and in red font indicate the dipeptide cleavage sites and variable amino acid residues around the cleavage sites, respectively; (B) Multiple sequence alignment of the P1 protein of four TeMV isolates.

Fig. 3 Evolutionary relationship and recombination pattern of telosma mosaic virus (TeMV). (A). Maximum-likelihood phylogenetic tree inferred from codon-aligned nucleotide sequences of the polyproteins of representative species of the genus Potyvirus, downloaded from GenBank. Three Rymoviruses (accession numbers: NC_005093, NC_005904 and NC_001814) were used as outgroups. Strong node support (UFboot support $>95 \%$ and SH-aLRT $>70 \%$ ) values are shown on each node. The five TeMV isolates are indicated in bold font, and Wuyishan and Fuzhou, TeMV isolates sequenced in this study are marked by black dots. (B). Graphical representation of recombinant regions in two TeMV isolates, Fuzhou and Wuyishan. (C). Recombination detection in TeMV genomes by using seven different algorithms implemented in RDP suite. NS, not significant.

Table S1 Primers used for RT-PCR and Rapid Amplification of cDNA Ends (RACE) in this study. 
Figure 1

Identification of $P$. edulis leaves infected with TeMV. 
Fig. 1

A.

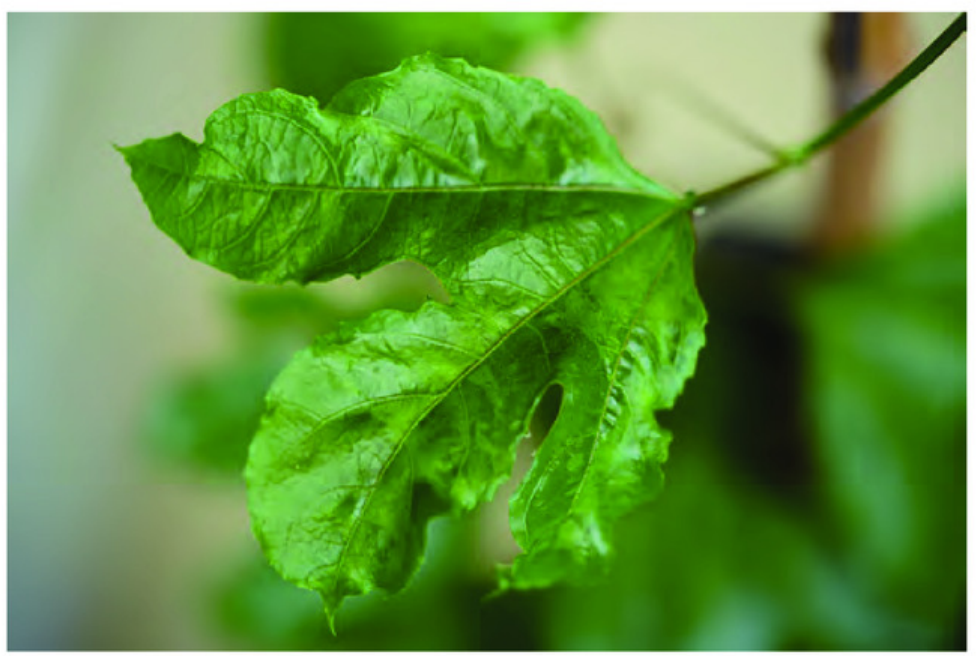

B.

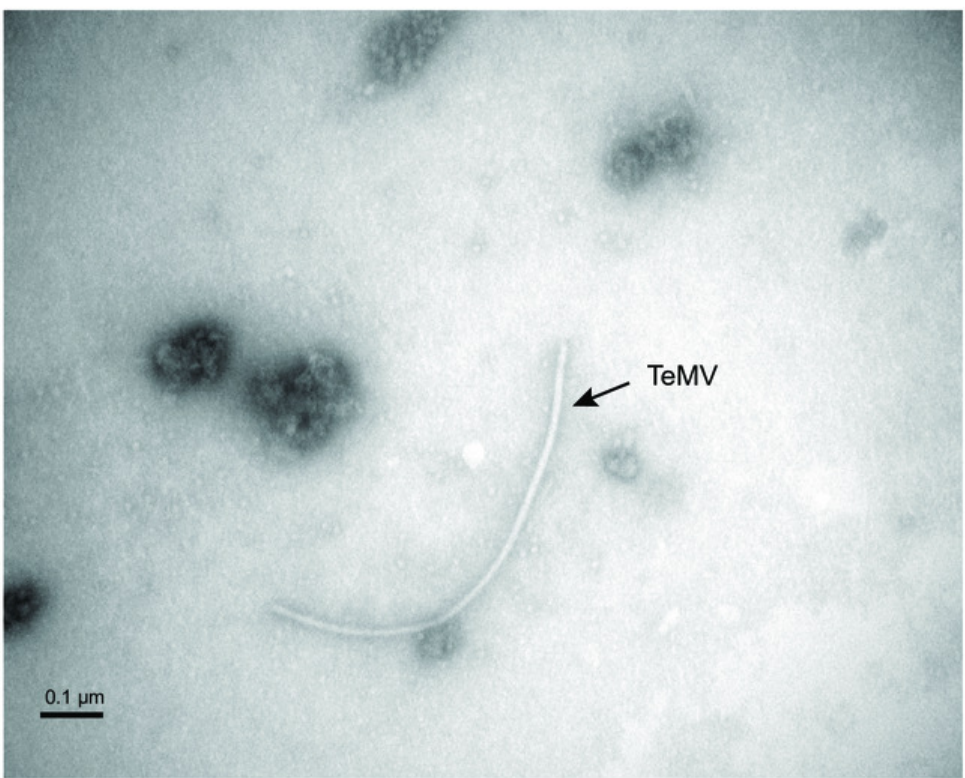

C.

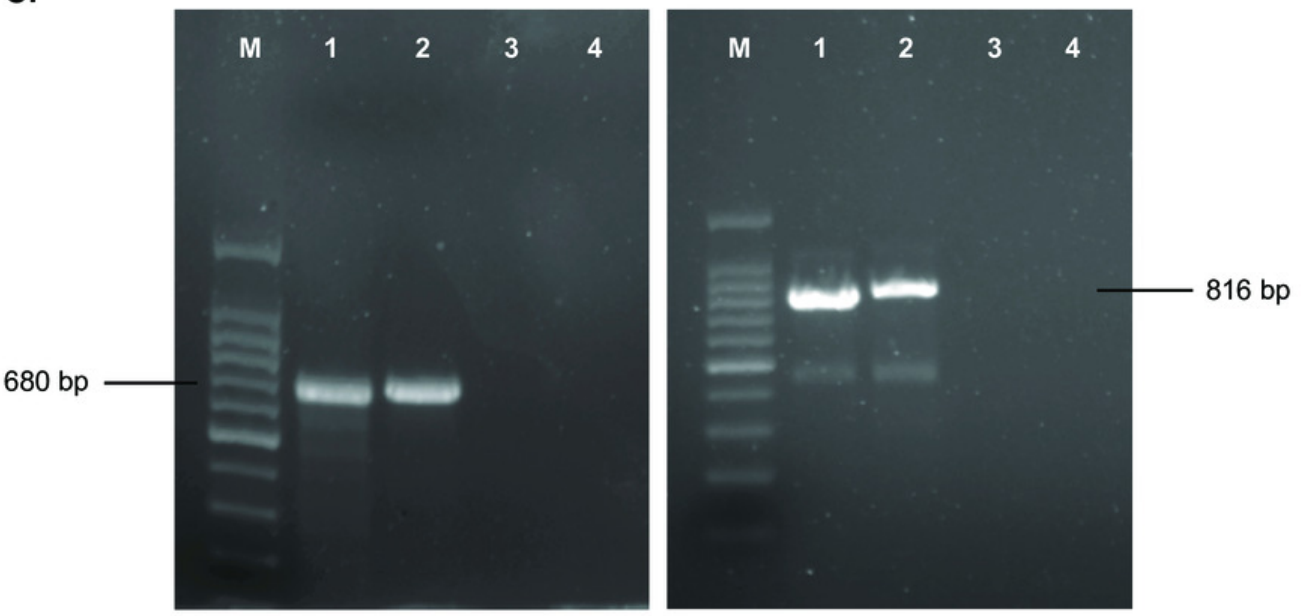


Figure 2

(A) Comparisons between the predicted protease cleavage sites of TeMV isolates; (B) Multiple sequence alignment of the P1 protein of four TeMV isolates. 
Fig. 2

A.

\begin{tabular}{|c|c|c|c|c|c|c|c|c|c|}
\hline & P1 & HC-Pro & P3 & $\mathrm{Cl}$ & $\mathrm{VPg}$ & $\mathrm{Nla}$ & Nlb & $\mathrm{CP}$ & Poly(A) \\
\hline $5^{2}$-UTR & & P3N-PIPO & $\circ$ & พี้ & y & & & & $3^{\prime}$-UTR \\
\hline Fuzhou & DVHHYIS & HYRVG/G & DVКTQ/G & TVRMO/S & FVkVE/S & AVKTQ/S & SVSLQ/S & & \\
\hline Wuyishan & EVHHYIS & HYRVG/G & DVQTQ/G & TVRMQ/S & FVKVE/S & AVKTQ/S & SVSLa/s & & \\
\hline GX & NA & NA & DVQTQ/G & TVRMQ/S & FVKVEIS & AVKTQ/S & SVSLQ/S & & \\
\hline PasFru & DVHHYIS & HYRVG/G & DVКTQ/G & TVRLQ/S & FVKVEIS & тVKTQ/S & SvSLa/s & & \\
\hline Hanoi & DVHHYIS & HYRVG/G & SVITQ/G & TVRLQ/S & RVTTE/S & TVKTQ/S & SVSLQ/S & & \\
\hline
\end{tabular}

B.
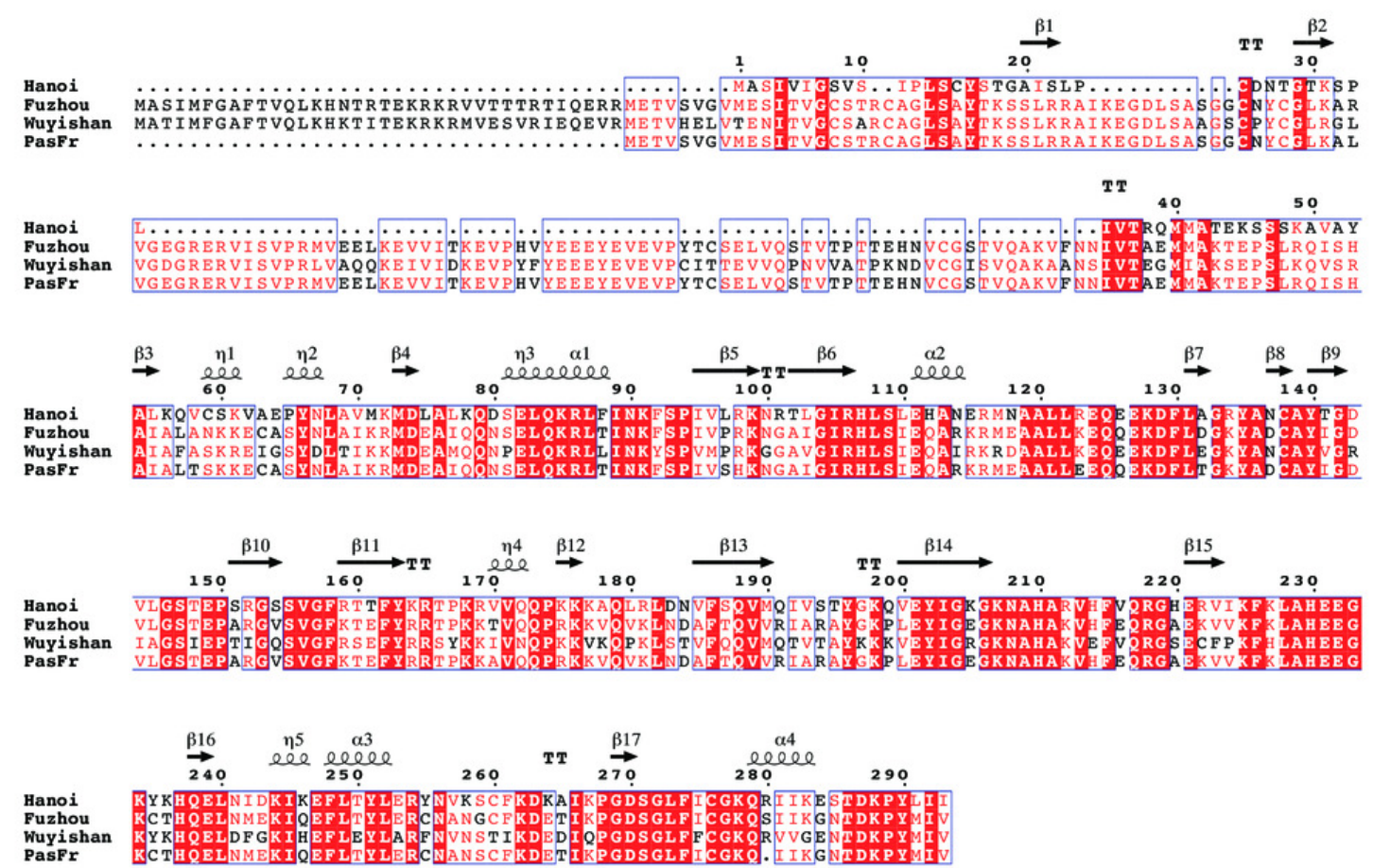
Figure 3

\section{Evolutionary relationship and recombination pattern of Telosma mosaic virus (TeMV).}

Fig. 3

A.

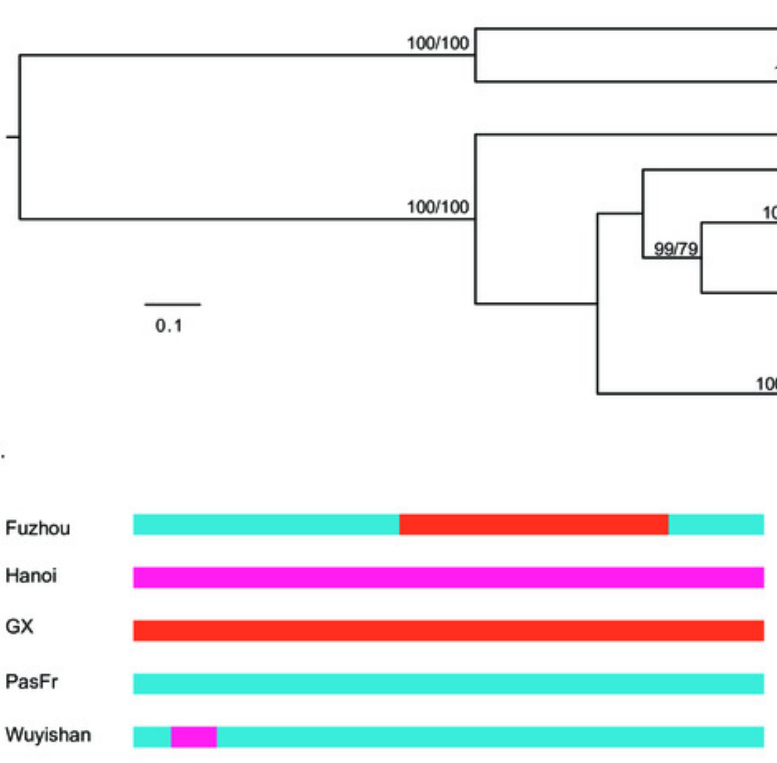

C.

\begin{tabular}{|c|c|c|c|c|c|c|c|}
\hline Recombinant & RDP & GENECONV & Bootscan & Maxchi & Chimaera & SiSscan & 3Seq \\
\hline Fuzhou & NS & $7.70 E-134$ & 1.34E-138 & 2.19E-45 & $5.65 E-47$ & $7.25 E-54$ & 4.44E-15 \\
\hline Wuyishan & 5.53E-06 & NS & $5.21 \mathrm{E}-23$ & 4.12E-18 & 2.31E-10 & $5.92 \mathrm{E}-09$ & 1.11E-14 \\
\hline
\end{tabular}

C_001814_RRGMB
C_005903_AgMV
C_005904_HoMV
C_009745_BBrMV
C_016159_KeuMV
C_002600_PeMoV
C_014064_FreMV
C_005304_BtMV
C_009741_BaRMV
C_003537_DsMV
C_003224_ZYMV
C_011560_ZMMV
C_014790_PaFrWoV
C_015394_HarMV
C_019415_BSVA
C_030236_IFBV
C_003397_BCMV
C_016441_YBMV
C_007728_EAPV
C_010954_FVV
C_007216_WVMV
C_002634_SMV
C_006262_WMV
C_021196_CLLV
C_004013_CAMV
C004047_BCMNV

